\title{
Morphometric evaluation and surgical implications of the infraorbital groove, canal and foramen on cone-beam computed tomography and a review of literature
}

\author{
i. Bahşi ${ }^{1}$, M. Orhan ${ }^{1}$, P. Kervancioğlu', E.D. Yalçin² \\ ${ }^{1}$ Department of Anatomy, Faculty of Medicine, Gaziantep University, Gaziantep, Turkey \\ 2Department of Dentomaxillofacial Radiology, Faculty of Dentistry, Gaziantep University, Gaziantep, Turkey \\ [Received: 25 June 2018; Accepted: 8 August 2018]
}

Background: The purpose of this study is to evaluate the anatomy, morphometry, and variations of infraorbital groove (IOG), infraorbital canal (IOC) and infraorbital foramen (IOF) on the cone-beam computed tomography (CBCT) images and to investigate their relations with surrounding structures.

Methods: IOG, IOC and IOF were evaluated retrospectively in CBCT images of 75 female (F) and 75 male $(M)$ cases with a range of 18-65 years ( $F$ : $37.62 \pm$ $\pm 13.55, \mathrm{M}: 37.53 \pm 15.87$ ) by Planmeca Romexis programme. IOG, IOC and IOF were examined bilaterally (300 sides) in the cases. The 13 parameters were measured on these images in axial, sagittal and coronal planes.

Results: There was a very weak positive correlation between the age and the angle between IOC and IOG ( $p=0.015, r=0.198)$, there was a weak positive correlation between the age and skin thickness $(p=0.001, r=0.281)$, and there was no correlation between the age and other parameters. A total of 21 (7\%) IOCs were detected in maxillary sinus, bilaterally in 6 cases and unilaterally in 9 cases (5 on the left, 4 on the right). In 1 case, bilaterally, IOC was separated 2 canals while running anteriorly in the maxillary sinus. The larger one was directed to IOF in its normal course and the smaller one was directed to lateral wall of nasal cavity and opened to the inferior nasal meatus in front of the opening of nasolacrimal duct. Conclusions: We suggest that the parameters found in the present study may facilitate prediction of the location of the infraorbital nerve. Knowledge of this exact position in relation to easily measurable parameters may decrease the risk of infraorbital nerve injury during surgical approaches directed to this region and might serve as a guide during local anaesthetic interventions for dentistry, ophthalmology, plastic surgery, rhinology, neurosurgery and dermatology. (Folia Morphol 2019; 78, 2: 331-343)

Key words: cone-beam computed tomography, infraorbital foramen, infraorbital canal, infraorbital groove, infraorbital nerve blockage

Address for correspondence: Dr. i. Bahşi, Department of Anatomy, Faculty of Medicine, Gaziantep University, Gaziantep, Turkey, tel: +90342 36060 60/74070, fax: +90 34247207 18, e-mail: dr.ilhanbahsi@gmail.com 


\section{INTRODUCTION}

Infraorbital groove (IOG), located on the inferior wall of the orbit and at the orbital surface of the body of maxilla, begin from the inferior orbital fissure posteriorly and continues as infraorbital canal (IOC) anteriorly and $\mathrm{IOC}$ opens to the middle of the face through the infraorbital foramen (IOF). Infraorbital nerve (ION) and vessels pass through IOG, IOC and spread from IOF [70, 74]. ION is responsible for the sensory innervation of upper cheek skin, maxillary sinus mucosa, maxillary incisor, canine and premolar teeth, occasionally mesiobuccal root of first molar tooth and adjacent buccolabial gingiva and periostium, the skin and conjunctiva of the inferior eyelid, part of the nose, and the skin and mucosa of the upper lip [52].

Anaesthesia of the ION may be performed on interventions such as surgical procedure of the facial region involving nose soft tissues, cheek, lower eyelid and maxillary premolar, canine and incisive teeth, orbital floor fracture treatment, nasal bone fracture reduction, scar revisions, cosmetic cutaneous procedures and polypectomy [51, 55, 74]. ION blockage may be performed for trigeminal neuralgia and postoperative pain $[2,27]$. It is known that ION paraesthesia may occur after surgical interventions [4,54]. Bilateral ION blockage is a preferred local method for early repair of cleft lip, facial lacerations, rhinoplasty repair and endoscopic endonasal maxillary sinus surgeries $[13,31,35,48,56]$. It is important to know the IOF neighbourhood for reducing complications in placement of malar, submalar, or paranasal implants [61]. The risk of ION's injury is high because it is difficult to determine the localisation and the course of the ION [4]. Although IOC is important, it is not clearly defined [79]. The pathway of ION is important for dentistry, ophthalmology, plastic surgery, rhinology, neurosurgery and dermatology. For this reason, it is substantial to know the morphometric properties of IOG, IOC, and IOF and their relations with surrounding structures.

The purpose of this study is to evaluate the anatomy, morphometry, and variations of IOG, IOC and IOF on the cone-beam computed tomography (CBCT) images and to investigate their relations with surrounding structures.

\section{MATERIALS AND METHODS}

Before the study, permission was received from the Clinical Trials Ethics Committee. A total of 150
CBCT images with no pathology of 75 female and 75 male subjects aged 18-65 years were selected randomly. The images of patients who were admitted to Gaziantep University Faculty of Dentistry for any reason were evaluated retrospectively by Planmeca Romexis (Planmeca, Helsinki, Finland) programme. On the images with artefact that prevents detection and measurement of reference points; maxillary sinus pathologies that may affect maxilla, IOG, IOC and IOF size; cases with developmental, metabolic or inflammatory jaw disease were not included in the study. IOG, IOC and IOF were examined bilaterally (300 sides) in the cases. The following 13 parameters were measured on these images in axial, sagittal and coronal planes (Fig. 1).

Axial:

-1 : The angle between the $\mathrm{IOC}$ and the sagittal plane (Fig. 1A)

Sagittal:

-2 : The angle between the $\mathrm{IOC}$ and the axial plane (Fig. 1B)

- 3: The angle between IOC and IOG (Fig. 1C)

- 4: $10 C$ length (Fig. 1C)

- 5: IOG length (Fig. 1C)

- 6: Vertical diameter of IOF (VD-IOF) (Fig. 1C)

- 7: The distance between IOF and infraorbital margin (IOF-IOM) (Fig. 1D)

- 8: Skin thickness over IOF (skin thickness) (ST) (Fig. 1D)

Coronal:

- 9: Transverse diameter of IOF (TD-IOF) (Fig. 1E)

- 10: The distance between IOF and the mid-sagittal plane (IOF-ML) (Fig. 1E)

- 11: The distance between IOF and the lateral wall of the nasal cavity (IOF-LNW) (Fig. 1F)

- 12: The distance between IOF and the occlusal plane of the second premolar tooth (IOF-PM). The distance between the transverse axis passing through the second premolar tooth level and the IOF was measured in the images where the IOF and the second premolar tooth were not in the same section (Fig. 1F)

- 13: The distance between IOF and vertical axis of lateral rim of frontozygomatic suture (IOF-FZS) (Fig. 1G, H)

\section{Statistical analysis}

The data were evaluated statistically. The normality of data distribution was tested by the Shapiro-Wilk test. 

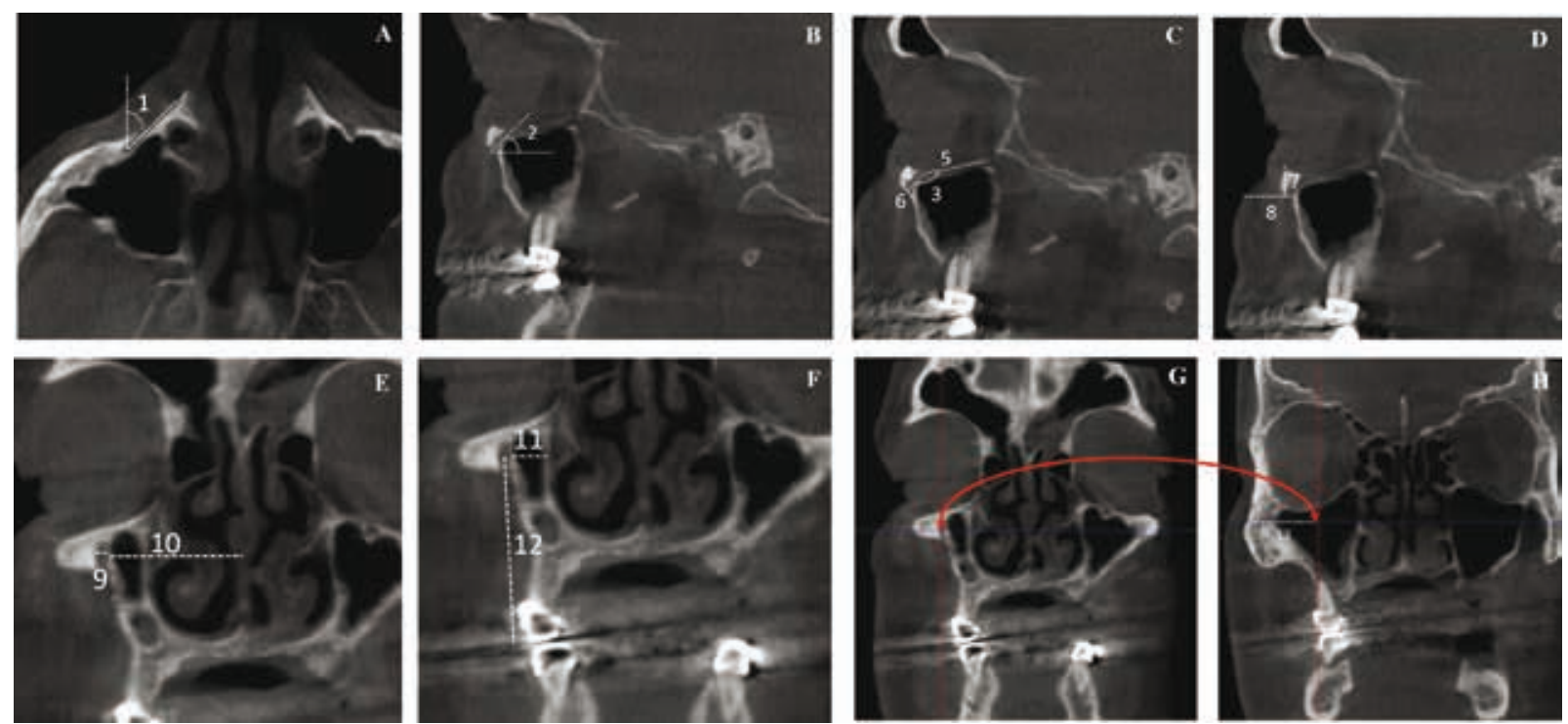

Figure 1. Thirteen parameters (abbreviations - see text) were measured on cone-beam computed tomography images in axial (A), sagittal (B, C, D) and coronal (E, F, G, H) planes.

Student $t$ test was used for comparison of variables with normal distribution in two independent groups, and paired $t$ test was used for comparison of two dependent measures. The Pearson correlation coefficient was used when the relations between numerical variables were tested. The SPSS 22.0 software package was used in the analyses and $p<0.05$ was accepted significant.

\section{RESULTS}

In this study, IOG, IOC and IOF were evaluated bilaterally in CBCT images of 75 female (F) and 75 male (M) cases with a range of 18-65 years (F: $37.62 \pm 13.55$, $M: 37.53 \pm 15.87$ ). No significant mean age difference existed between genders $(p>0.956)$. Thirteen parameters were evaluated (Table 1). The parameters were compared by gender; significant differences were found in the IOC length (right $[R]: p=0.029$, left [L]: $p=0.021)$, in the IOF-IOM (R: $p=0.005, L: p=0.012)$ and in the VD-IOF (R: $p=0.001, L: p=0.002$ ).

There was a very weak positive correlation between the age and the angle between IOC and IOG $(p=0.015, r=0.198)$, there was also a weak positive correlation between the age and ST ( $p=0.001$, $r=0.281$ ), and there was no correlation between the age and other parameters.

A total of $21(7 \%)$ IOCs were detected in maxillary sinus, bilaterally in 6 cases, and unilaterally in 9 cases (5 on the left, 4 on the right) (Fig. 2).

In 1 case, bilaterally, IOC was divided into 2 canals while running anteriorly in the maxillary sinus. The larger one was directed to IOF in its normal course and the smaller one was directed to lateral wall of nasal cavity and opened to the inferior nasal meatus in front of the opening of nasolacrimal duct (Fig. 3).

\section{DISCUSSION}

There are many bone $[2,12,18,20,38,45,53,57$, $58,60,66,68]$ and cadaver $[6,14,25,39,44,59,66$, 71] studies in which the relationship between morphometry of IOG, IOC, and IOF and their relations to the surrounding structures have been examined. Kazkayasi et al. [38] evaluated 35 dry skulls both in bones and cephalometric radiography. Lee et al. [43] evaluated the computed tomography (CT) of the 42 dry skulls. In the literature, there are publications in which IOG, IOC or IOF are assessed with $C T$ in the living person $[34,36,42$, $61,67,72,78,79]$. There are few publications in which these three structures have been evaluated like in this study [34]. CT can show localisation and morphometry of the structures in three dimensions. Hwang et al. [34] stated that CT mediated measurements were as effective as dry skull and cadaver measurements. The most important advantage of $\mathrm{CT}$ measurements in the living person compared to dry skull and cadaver is that the gender and age of the cases are known precisely. In recent years, the use of CBCT with cheap cost and low radiation doses has increased for dentomaxillofacial diagnosis and treatment $[7,30]$.

Eppley [24] stated that at the ION injury may result in dysesthesia. Vriens et al. [77] stated long term sensory disturbances vary between $24 \%$ and $50 \%$ after orbitozygomatic complex traumas. ION localisation 
Table 1. The measurements of 13 parameters about infraorbital groove (IOG), infraorbital canal (IOC) and infraorbital foramen (IOF)

\begin{tabular}{|c|c|c|c|c|}
\hline Parameter & Gender & Right & Left & $\mathbf{P}$ \\
\hline \multirow{3}{*}{ 1. The angle between the $I O C$ and the sagittal plane $\left[^{\circ}\right]$} & $\mathrm{F}$ & $35.38 \pm 7.77$ & $35.42 \pm 8.49$ & 0.839 \\
\hline & $\mathrm{M}$ & $34.23 \pm 8.60$ & $33.44 \pm 9.07$ & 0.154 \\
\hline & Total & $34.81 \pm 8.19$ & $34.44 \pm 8.81$ & 0.215 \\
\hline \multirow{3}{*}{ 2. The angle between the $\mathrm{IOC}$ and the axial plane $\left[^{\circ}\right]$} & $\mathrm{F}$ & $46.81 \pm 6.82$ & $46.41 \pm 7.54$ & 0.580 \\
\hline & $\mathrm{M}$ & $47.58 \pm 7.34$ & $47.22 \pm 7.71$ & 0.665 \\
\hline & Total & $47.20 \pm 7.07$ & $46.81 \pm 7.61$ & 0.489 \\
\hline \multirow{3}{*}{ 3. The angle between IOC and IOG [ $\left.{ }^{\circ}\right]$} & $\mathrm{F}$ & $144.77 \pm 7.29$ & $144.82 \pm 7.93$ & 0.961 \\
\hline & $\mathrm{M}$ & $143.40 \pm 6.41$ & $143.22 \pm 6.88$ & 0.856 \\
\hline & Total & $144.08 \pm 6.41$ & $144.02 \pm 7.44$ & 0.925 \\
\hline \multirow{3}{*}{ 4. IOC length [mm] } & $\mathrm{F}$ & $8.20 \pm 1.60$ & $8.45 \pm 1.94$ & 0.244 \\
\hline & $\mathrm{M}$ & $8.37 \pm 1.78$ & $8.45 \pm 1.80$ & 0.654 \\
\hline & Total & $8.28 \pm 1.69$ & $8.45 \pm 1.87$ & 0.233 \\
\hline \multirow{3}{*}{ 5. IOG length $[\mathrm{mm}]$} & $\mathrm{F}$ & $21.90 \pm 3.57$ & $20.49 \pm 3.49$ & $0.001^{*}$ \\
\hline & $\mathrm{M}$ & $23.28 \pm 4.07$ & $21.97 \pm 4.29$ & $0.004^{*}$ \\
\hline & Total & $22.59 \pm 3.88$ & $21.23 \pm 3.97$ & $0.001^{*}$ \\
\hline \multirow{3}{*}{ 6. Vertical diameter of IOF (VD-IOF) [mm] } & $\mathrm{F}$ & $3.21 \pm 0.45$ & $3.11 \pm 0.55$ & 0.194 \\
\hline & $\mathrm{M}$ & $3.77 \pm 1.30$ & $3.46 \pm 0.80$ & $0.034^{*}$ \\
\hline & Total & $3.49 \pm 1.01$ & $3.29 \pm 0.71$ & $0.013^{*}$ \\
\hline \multirow{3}{*}{ 7. The distance between IOF and infraorbital margin (IOF-IOM) [mm] } & $\mathrm{F}$ & $7.15 \pm 1.22$ & $7.10 \pm 1.30$ & 0.714 \\
\hline & $\mathrm{M}$ & $7.79 \pm 1.49$ & $7.67 \pm 1.47$ & 0.368 \\
\hline & Total & $7.47 \pm 1.40$ & $7.39 \pm 1.41$ & 0.384 \\
\hline \multirow{3}{*}{ 8. Skin thickness over IOF (skin thickness) (ST) [mm] } & $\mathrm{F}$ & $9.99 \pm 2.48$ & $10.17 \pm 2.38$ & 0.303 \\
\hline & $\mathrm{M}$ & $9.31 \pm 1.64$ & $9.45 \pm 1.95$ & 0.454 \\
\hline & Total & $9.65 \pm 2.13$ & $9.81 \pm 2.19$ & 0.208 \\
\hline \multirow{3}{*}{ 9. Transvers diameter of IOF (TD-IOF) [mm] } & $\mathrm{F}$ & $3.32 \pm 0.47$ & $3.15 \pm 0.45$ & $0.001^{*}$ \\
\hline & $\mathrm{M}$ & $3.42 \pm 0.56$ & $3.29 \pm 0.66$ & 0.147 \\
\hline & Total & $3.37 \pm 0.52$ & $3.22 \pm 0.57$ & $0.004^{*}$ \\
\hline \multirow{3}{*}{ 10. The distance between IOF and the mid-sagittal plane (IOF-ML) [mm] } & $\mathrm{F}$ & $22.65 \pm 2.17$ & $22.67 \pm 2.39$ & 0.922 \\
\hline & $\mathrm{M}$ & $24.39 \pm 2.00$ & $24.19 \pm 2.15$ & 0.466 \\
\hline & Total & $23.52 \pm 2.26$ & $23.43 \pm 2.39$ & 0.640 \\
\hline \multirow{3}{*}{$\begin{array}{l}\text { 11. The distance between IOF and the lateral wall of the nasal cavity } \\
\text { (IOF-LNW) [mm] }\end{array}$} & $\mathrm{F}$ & $9.24 \pm 2.34$ & $8.77 \pm 2.19$ & $0.026^{*}$ \\
\hline & $\mathrm{M}$ & $9.89 \pm 2.42$ & $9.90 \pm 2.41$ & 0.966 \\
\hline & Total & $9.57 \pm 2.39$ & $9.34 \pm 2.36$ & 0.154 \\
\hline \multirow{3}{*}{$\begin{array}{l}\text { 12. The distance between IOF and the occlusal plane of the second } \\
\text { premolar tooth (IOF-PM) [mm] }\end{array}$} & $\mathrm{F}$ & $38.04 \pm 1.75$ & $37.94 \pm 1.96$ & 0.122 \\
\hline & $\mathrm{M}$ & $39.33 \pm 2.99$ & $39.94 \pm 3.06$ & 0.110 \\
\hline & Total & $38.68 \pm 2.53$ & $38.94 \pm 2.75$ & 0.102 \\
\hline \multirow{3}{*}{$\begin{array}{l}\text { 13. The distance between IOF and vertical axis of lateral rim of } \\
\text { frontozygomatic suture (IOF-FZS) [mm] }\end{array}$} & $\mathrm{F}$ & $24.68 \pm 1.45$ & $24.59 \pm 1.38$ & 0.161 \\
\hline & $\mathrm{M}$ & $24.40 \pm 1.22$ & $24.47 \pm 1.37$ & 0.612 \\
\hline & Total & $24.54 \pm 1.34$ & $24.53 \pm 1.34$ & 0.829 \\
\hline
\end{tabular}

*Significant difference; $\mathrm{F}$ - female; $\mathrm{M}$ - male
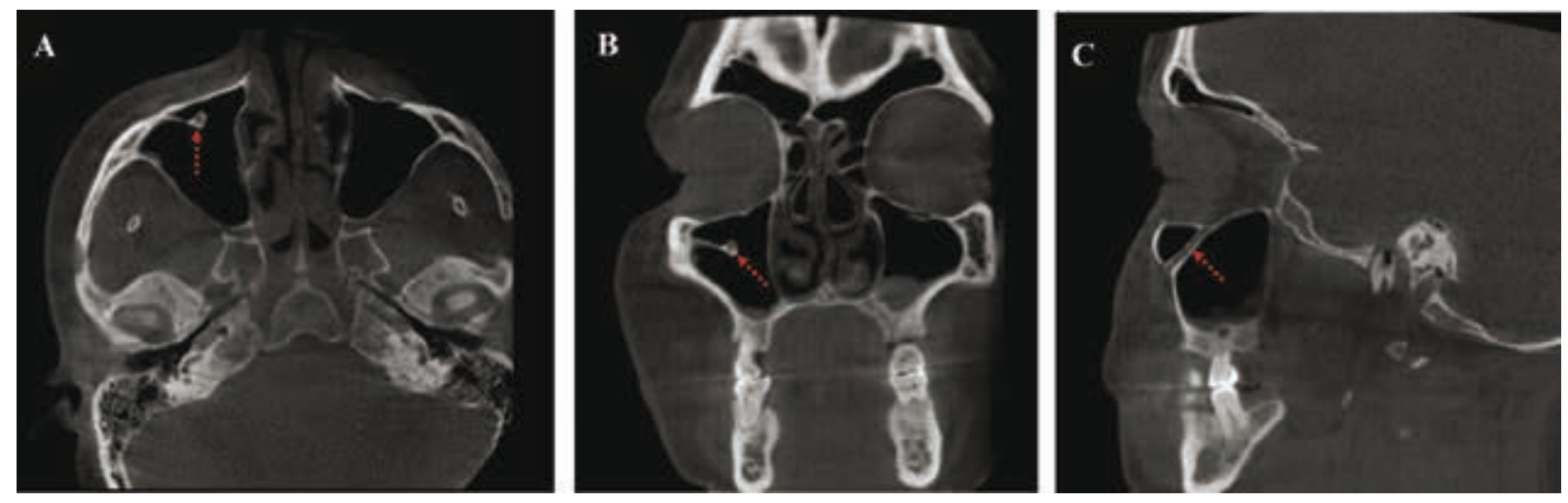

Figure 2. Infraorbital canal was detected in the maxillary sinus; A. Axial plane; B. Coronal plane; C. Sagittal plane. 

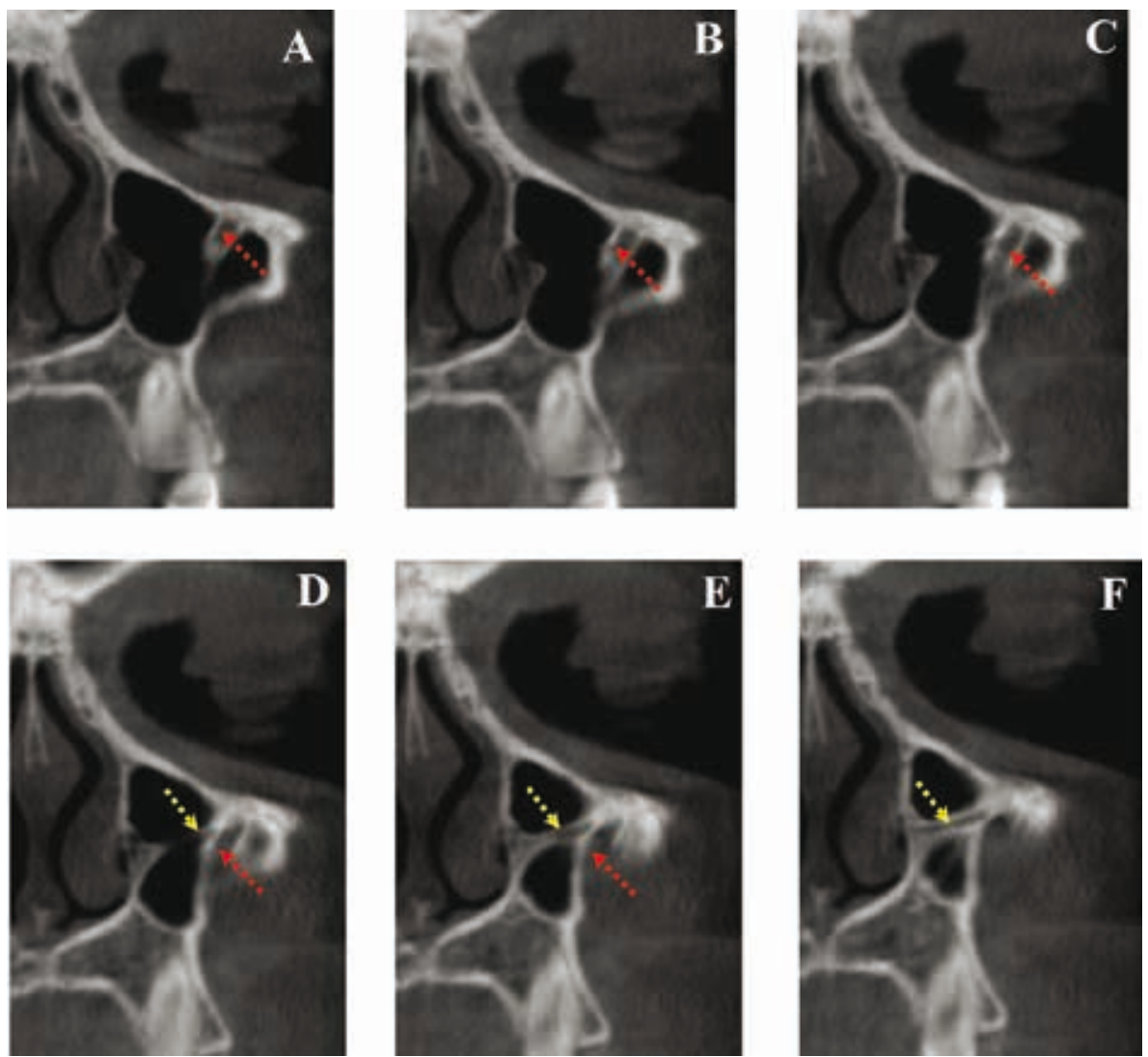

Figure 3. Infraorbital canal (IOC) was divided into two canals while running from posterior to anterior in the maxillary sinus (red arrow: the larger one, yellow arrow: the smaller one).

should be kept in mind in maxilla, zygoma, or deep cheek injury procedures [33]. A common description of the radiofrequency neurotomy procedure for ION is not available. The angle between the IOC and the sagittal plane, the angle between the IOC and the axial plane, IOF-ML and IOF-IOM distances should be known for targeting the needle to IOF and $\mathrm{IOC}$ applying the radiofrequency neurotomy [59]. ST is also important during the intervention. In addition, advancing the needle behind the IOC may damage structures in the orbital cavity or the pterygopalatine fossa [34]. Saeedi et al. [65] and Chan et al. [15] reported cases of blunt eye injury as a complication of ION blockade. The shorter and steeper the $I O C$ is, the more likely it is that the eyeball will be injured. Therefore, it is important to know morphometry of IOG, IOC and IOF that the ION passes through.

The angle between the $10 \mathrm{C}$ and the sagittal plane. In the studies performed [2, 34, 43, 59, 78], results were found in a wide range between $12^{\circ}$ and $69^{\circ}$ and in our study this angle was found as R: $34.81^{\circ}$, L: $34.44^{\circ}$ (Table 2).
The angle between the $\mathrm{IOC}$ and the axial plane. It has been noted that in studies evaluating $\mathrm{CT}$ images $[34,78]$ this angle gives higher values than dry skull $[2,3,43]$ and cadaver studies [59] (Table 2). Hwang et al. [34] stated that this angle was statistically significantly different between the genders $(p=0.038)$. In our study, there was no statistically significant difference between the genders (Table 2).

The angle between IOC and IOG. Only Hwang et al. [34] reported measuring this angle in the literature. Similar result was found in our study (Table 2).

IOC and IOG lengths. Frequently examined in dry skull $[32,38,58,63]$ and cadaver studies $[49,59]$, and less frequently in CT images $[34,78]$. In studies evaluating $\mathrm{CT}$ images including the present study, IOC length was shorter than in dry skull and cadaver studies and IOG length was longer. The bony part which forms the upper wall of the back of the IOC is so thin that cannot be clearly detected in the CT images, which may be the possible reason of these differences. Present study and Hwang et al. [34] found a significant difference in IOG length between the genders ( $p=0.001, p=0.012$, respectively) (Table 3 ). 
Table 2. Comparison of the angle between infraorbital canal (IOC) and the sagittal plane, IOC and the axial plane, IOC and infraorbital groove (IOG) with the literature

\begin{tabular}{|c|c|c|c|c|c|c|c|c|c|c|c|}
\hline \multirow[t]{2}{*}{ Study } & \multirow[t]{2}{*}{ Specimen } & \multirow[t]{2}{*}{ Side } & \multicolumn{3}{|c|}{$\begin{array}{l}\text { The angle between the } \mathrm{IOC}^{\circ} \\
\text { and the sagittal plane }\left[^{\circ}\right]\end{array}$} & \multicolumn{3}{|c|}{$\begin{array}{c}\text { The angle between the IOC } \\
\text { and the axial plane }\left[^{\circ}\right]\end{array}$} & \multicolumn{3}{|c|}{$\begin{array}{c}\text { The angle between IOC } \\
\text { and IOG [०] }\end{array}$} \\
\hline & & & $\mathbf{F}$ & M & Total & $F$ & $\mathbf{M}$ & Total & $\mathbf{F}$ & M & Total \\
\hline \multirow[t]{2}{*}{ Aggarwal et al. [2] } & \multirow[t]{2}{*}{ Dry skull } & $\mathrm{R}$ & \multicolumn{2}{|c|}{$21.48 \pm 10.41$} & \multirow{2}{*}{$\begin{array}{c}21.14 \pm \\
10.10\end{array}$} & \multicolumn{2}{|c|}{$31.43 \pm 7.97$} & \multirow{2}{*}{$\begin{array}{c}31.79 \pm \\
7.68\end{array}$} & & & \\
\hline & & L & 20.81 & +9.92 & & 32.10 & $=7.43$ & & & & \\
\hline \multirow[t]{2}{*}{ Rahman et al. [59] } & \multirow[t]{2}{*}{ Cadaver } & $\mathrm{R}$ & & & \multirow{2}{*}{22} & & & \multirow{2}{*}{30} & & & \\
\hline & & $L$ & & & & & & & & & \\
\hline \multirow[t]{2}{*}{ Lee et al. [43] } & \multirow[t]{2}{*}{ Dry skull } & $\mathrm{R}$ & & & \multirow{2}{*}{$12 \pm 6.5$} & & & \multirow{2}{*}{$44 \pm 7.9$} & & & \\
\hline & & $L$ & & & & & & & & & \\
\hline \multirow[t]{2}{*}{ Agthong et al. [3] } & \multirow[t]{2}{*}{ Dry skull } & $\mathrm{R}$ & & & & $24.4 \pm 0.7$ & $25.4 \pm 0.5$ & $25.1 \pm 0.4$ & & & \\
\hline & & L & & & & $26.3 \pm 0.6$ & $27.0 \pm 0.6$ & $26.8 \pm 0.4$ & & & \\
\hline \multirow[t]{2}{*}{ Hwang et al. [34] } & \multirow[t]{2}{*}{ HRCT } & $\mathrm{R}$ & \multirow{2}{*}{$\begin{array}{c}13.0 \pm \\
6.5\end{array}$} & \multirow{2}{*}{$\begin{array}{c}13.5 \pm \\
6.3\end{array}$} & \multirow{2}{*}{$\begin{array}{c}13.2 \pm \\
6.4\end{array}$} & \multirow{2}{*}{$\begin{array}{c}47.6 \pm \\
7.6^{*}\end{array}$} & \multirow{2}{*}{$\begin{array}{c}45.3 \pm \\
7.4^{*}\end{array}$} & \multirow{2}{*}{$\begin{array}{c}46.7 \pm \\
7.6^{*}\end{array}$} & \multirow{2}{*}{$\begin{array}{c}144.8 \pm \\
9.0\end{array}$} & \multirow{2}{*}{$\begin{array}{c}146.8 \pm \\
7.4\end{array}$} & \multirow{2}{*}{$\begin{array}{c}145.5 \pm \\
8.5\end{array}$} \\
\hline & & $L$ & & & & & & & & & \\
\hline \multirow[t]{2}{*}{ Xu et al. [78] } & \multirow[t]{2}{*}{ CT } & $\mathrm{R}$ & $\begin{array}{c}67.96 \pm \\
10.01\end{array}$ & $\begin{array}{c}69.22 \pm \\
8.52 \\
\end{array}$ & & $\begin{array}{c}65.72 \pm \\
6.82 \\
\end{array}$ & $\begin{array}{c}68.25 \pm \\
6.97\end{array}$ & & & & \\
\hline & & $L$ & $\begin{array}{c}67.30 \pm \\
10.08\end{array}$ & $\begin{array}{c}68.71 \pm \\
9.19\end{array}$ & & $\begin{array}{c}66.19 \pm \\
6.28 \\
\end{array}$ & $\begin{array}{l}68.22 \pm \\
7.15\end{array}$ & & & & \\
\hline Present study & СВСТ & $\mathbf{R}$ & $\begin{array}{c}35.38 \pm \\
7.77\end{array}$ & $\begin{array}{c}34.23 \pm \\
8.60\end{array}$ & $\begin{array}{c}34.81 \pm \\
8.19\end{array}$ & $\begin{array}{l}46.81 \pm \\
6.82\end{array}$ & $\begin{array}{c}47.58 \pm \\
7.34\end{array}$ & $\begin{array}{c}47.20 \pm \\
7.07\end{array}$ & $\begin{array}{l}144.77 \\
\pm 7.29\end{array}$ & $\begin{array}{l}143.40 \\
\pm 6.41\end{array}$ & $\begin{array}{l}144.08 \\
\pm 6.41\end{array}$ \\
\hline & & $\mathbf{L}$ & $\begin{array}{c}35.42 \pm \\
8.49\end{array}$ & $\begin{array}{c}33.44 \pm \\
9.07\end{array}$ & $\begin{array}{c}34.44 \pm \\
8.81\end{array}$ & $\begin{array}{c}46.41 \pm \\
7.54\end{array}$ & $\begin{array}{c}47.22 \pm \\
7.71\end{array}$ & $\begin{array}{c}46.81 \pm \\
7.61\end{array}$ & $\begin{array}{l}144.82 \\
\pm 7.93\end{array}$ & $\begin{array}{l}143.22 \\
\pm 6.88\end{array}$ & $\begin{array}{l}144.02 \\
\pm 7.44\end{array}$ \\
\hline
\end{tabular}

${ }^{*}$ Significant difference; L — left; R — right; F — female; M — male; CBCT — cone-beam computed tomography; CT — computed tomography; HRCT — high resolution computed tomography

VD-IOF and TD-IOF. These are measured in a number of publications in the literature $[2,5,6,10,12$, $18-20,36,40,68,69,71-73$ ]. Kara et al. [36] found the mean diameter of the IOF and found a significant difference between the genders $(p=0.001)$. Cisneiros de Oliveira et al. [20] reported a significant difference in left VD-IOF, right TD-IOF and left TD-IOF measurements between the genders, as well as VDIOF measurements between the both sides. In our study, there was a significant difference in VD-IOF and TD-IOF measurements between the both sides ( $p=0.013, p=0.004$, respectively) (Table 4).

Distance between IOF-IOM. The most measured distance $[1-3,5,6,12,18,20-22,25,28,29$, $34,37,38,42,45,46,50,59,61,62,66,68,72$, $73,75,78]$. This distance is used for the detection of IOF localisation in extraoral ION blockade approach and radiofrequency neurotomy. CT measurements show relatively higher values than dry skull measurements. Kazkayasi et al. [38] measured $7.19 \pm$ $\pm 1.39 \mathrm{~mm}$ in the dry skull, and $7.45 \pm 0.95 \mathrm{~mm}$ in the X-ray graph. By a cadaveric study, Cutright et al. [21] found $5.80 \pm 0.30 \mathrm{~mm}$ in white females, $7.10 \pm 0.30 \mathrm{~mm}$ in white males, $5.70 \pm 0.20 \mathrm{~mm}$ in black females and $6.90 \pm 0.30 \mathrm{~mm}$ in black males. Apinhasmit et al. [5] reported the significant difference in IOF-IOM distance between the genders. Elias et al. [22] and Macedo et al. [46] reported there was a significant difference in IOF-IOM distance between the both sides. In this study, it was observed that the distance between IOF-IOM was similar to other studies and there was no significant difference between the sides and genders (Table 5).

ST length. Hwang et al. [34] determined the ST length as $11.4 \mathrm{~mm}$. In this study, length was determined as R: $9.65 \mathrm{~mm}$ and L: $9.81 \mathrm{~mm}$ (Table 6). ST length is important in extraoral ION blockade approach and radiofrequency neurotomy. For this reason, it is appropriate to move the needle 9-11 $\mathrm{mm}$ from the skin surface to reach the IOF.

The distance between IOF-ML. It is determined between 25.20-30.30 mm [2, 5, 18, 25, 29, 34, 59, 72,78 ] (Table 5). In our study, the measurement was relatively shorter when compared to the literature (R: $23.52 \mathrm{~mm}$, L: $23.43 \mathrm{~mm}$ ) (Table 5).

Distance between IOF-LNW or IOF-piriform aperture (IOF-PA). The distance between the IOF-PA examined on spiral CT images [61], skull [2, 18, 22, 38, 
Table 3. Comparison of infraorbital canal (IOC), infraorbital groove (IOG) and IOC + IOG lengths with the literature

\begin{tabular}{|c|c|c|c|c|c|c|c|c|c|}
\hline \multirow[t]{2}{*}{ Study } & \multirow[t]{2}{*}{ Specimen } & \multirow[t]{2}{*}{ Side } & \multicolumn{3}{|c|}{ IOC length [mm] } & \multicolumn{3}{|c|}{ IOG length [mm] } & \multirow{2}{*}{$\frac{\mathrm{IOC}+\mathrm{IOG} \text { length }[\mathrm{mm}]}{\text { Total }}$} \\
\hline & & & $\mathbf{F}$ & M & Total & $\mathbf{F}$ & M & Total & \\
\hline \multirow{2}{*}{$\begin{array}{l}\text { Hwang et al. } \\
\text { [34] }\end{array}$} & \multirow[t]{2}{*}{ HRCT } & $\mathrm{R}$ & & & \multirow{2}{*}{$11.7 \pm 1.9$} & \multirow{2}{*}{\multicolumn{3}{|c|}{$16.7 \pm 2.4$ * }} & \\
\hline & & $\mathrm{L}$ & & & & & & & \\
\hline \multirow{2}{*}{$\begin{array}{l}\text { Kazkayası et al. } \\
{[38]}\end{array}$} & \multirow[t]{2}{*}{ Dry skull } & $\mathrm{R}$ & & & \multirow{2}{*}{$22.95 \pm 5.43$} & \multirow{2}{*}{\multicolumn{3}{|c|}{$5.95 \pm 4.90$}} & \\
\hline & & $\mathrm{L}$ & & & & & & & \\
\hline \multirow{2}{*}{$\begin{array}{l}\text { Pryzgocka et al. } \\
\text { [58] }\end{array}$} & \multirow[t]{2}{*}{ Dry skull } & $\mathrm{R}$ & & & $14.23 \pm 4.68$ & & & $13.49 \pm 3.87$ & $27.71 \pm 3.54$ \\
\hline & & $\mathrm{L}$ & & & $13.71 \pm 4.62$ & & & $14.14 \pm 4.36$ & $28.11 \pm 3.22$ \\
\hline \multirow{2}{*}{$\begin{array}{l}\text { Huanmanop et al. } \\
\text { [32] }\end{array}$} & \multirow[t]{2}{*}{ Dry skull } & $\mathrm{R}$ & $13.2 \pm 4.4$ & $11.3 \pm 3.6$ & $12.3 \pm 4.1$ & & & & \\
\hline & & $\mathrm{L}$ & $12.5 \pm 3.5$ & $12.3 \pm 3.4$ & $12.4 \pm 3.4$ & & & & \\
\hline \multirow{2}{*}{$\begin{array}{l}\text { McQueen et al. } \\
\text { [49] }\end{array}$} & \multirow[t]{2}{*}{ Cadaver } & $\mathrm{R}$ & & & \multirow{2}{*}{$17.08 \pm 3.64$} & & & & \\
\hline & & $\mathrm{L}$ & & & & & & & \\
\hline \multirow{2}{*}{$\begin{array}{l}\text { Rontal et al. } \\
\text { [63] }\end{array}$} & \multirow[t]{2}{*}{ Dry skull } & $\mathrm{R}$ & & & \multirow{2}{*}{$14(8-28)$} & & & & \\
\hline & & $\mathrm{L}$ & & & & & & & \\
\hline \multirow{2}{*}{$\begin{array}{l}\text { Xu et al. } \\
\text { [78] }\end{array}$} & \multirow[t]{2}{*}{ CT } & $\mathrm{R}$ & $9.52 \pm 3.00$ & $10.29 \pm 2.57$ & $9.98 \pm 2.75$ & & & & \\
\hline & & $\mathrm{L}$ & $9.48 \pm 2.71$ & $10.18 \pm 2.31$ & $9.92 \pm 2.47$ & & & & \\
\hline \multirow{2}{*}{$\begin{array}{l}\text { Rahman et al. } \\
\text { [59] }\end{array}$} & \multirow[t]{2}{*}{ Cadaver } & $\mathrm{R}$ & & & \multirow{2}{*}{14} & \multirow{2}{*}{\multicolumn{3}{|c|}{13}} & \\
\hline & & $\mathrm{L}$ & & & & & & & \\
\hline \multirow{2}{*}{$\begin{array}{l}\text { Berge and } \\
\text { Bergman [10] }\end{array}$} & Dry skull & $\mathrm{R}$ & & & & & & & 2770 \\
\hline & & $\mathrm{L}$ & & & & & & & 21.10 \\
\hline Karakas et al. & Dry skull & $\mathrm{R}$ & & & & & & & $310+30$ \\
\hline & & $\mathrm{L}$ & & & & & & & J1.9 \\
\hline Abed et al. & Cadaver & $\mathrm{R}$ & & & & & & & $254+27$ \\
\hline & & $\mathrm{L}$ & & & & & & & $20.4-2.1$ \\
\hline Present study & СВСТ & $\mathbf{R}$ & $\begin{array}{c}8.20 \pm \\
1.60\end{array}$ & $\begin{array}{c}8.37 \pm \\
1.78\end{array}$ & $\begin{array}{c}8.28 \pm \\
1.69\end{array}$ & $\begin{array}{c}21.90 \pm \\
3.57^{*}\end{array}$ & $\begin{array}{c}23.28 \pm \\
4.07^{*}\end{array}$ & $\begin{array}{c}22.59 \pm \\
3.88^{*}\end{array}$ & \\
\hline & & $\mathbf{L}$ & $\begin{array}{c}8.45 \pm \\
1.94\end{array}$ & $\begin{array}{c}8.45 \pm \\
1.80\end{array}$ & $\begin{array}{c}8.45 \pm \\
1.87\end{array}$ & $\begin{array}{c}20.49 \pm \\
3.49^{*}\end{array}$ & $\begin{array}{c}21.97 \pm \\
4.29 *\end{array}$ & $\begin{array}{c}21.23 \pm \\
3.97^{*}\end{array}$ & \\
\hline
\end{tabular}

*Significant difference; L — left; R — right; F — female; M — male; CBCT — cone-beam computed tomography; CT — computed tomography; HRCT — high resolution computed tomography

$45,46,68,75$ ] and cadaver [59]. IOF-LNW examined on cephalometric radiographs [38], MD-CT images [72]. This is the first study, in which the distance between IOF-LNW was evaluated with CBCT. Elias et al. [22] pointed out that the distance between IOF-PA was statistically significant between the both sides. In the present study, the IOF-LNW distance was relatively shorter (R: $9.57 \pm 2.39 \mathrm{~mm}, \mathrm{~L}: 9.34 \pm 2.36 \mathrm{~mm})$ than reported in the previous studies (Table 7).

Distance between IOF-PM. Taşpınar [72] measured the distance between the root of second incisive tooth and IOF (R: $29.97 \pm 3.10 \mathrm{~mm}, \mathrm{~L}: 30.20 \pm$ $\pm 3.33 \mathrm{~mm}$ ), Kara et al. [36] measured the distance between the IOF and the lateral process of canine tooth (F: $37.10 \pm 5.30 \mathrm{~mm}, \mathrm{M}: 34.50 \pm 4.60 \mathrm{~mm}$ ). Kazkayasi et al. [38] stated IOF to the lateral process of the canine tooth in the vertical direction was $33.94 \pm$ $\pm 3.15 \mathrm{~mm}$. Ukoha et al. [75] and Gour et al. [28] stated IOF was detected most frequently at the level of the second premolar teeth $(46 \%, 43 \%$, respectively). For this reason, IOF-PM distance in the coronal plane was measured in this study. Raschke et al. [61] determined the IOF-PM distance $41.81 \pm 1.07 \mathrm{~mm}$ in males and $37.23 \pm 1.58$. in females. In the present study, similar measurements were found with Raschke et al. [61], $38.68 \pm 2.53 \mathrm{~mm}$ on the right side and $38.94 \pm 2.75 \mathrm{~mm}$ on the left side (Table 6).

Distance between IOF-FZS. Raschke et al. [61] assessed the distance between the lateral orbital rim and IOF (Table 7). In this study, the distance between IOF and vertical axis of lateral rim of frontozygomatic suture was measured, because it was more precisely detected than the lateral orbital rim on the CBCT images. The results obtained in both studies are similar to each other (24-25 mm) (Table 6). This distance will be clinically useful for identifying IOF localisation.

There is little in literature about variation related to IOC. Chandra and Kennedy [16], Elnil et al. [23] 
Table 4. Comparison of diameters of infraorbital foramen (IOF) with the literature

\begin{tabular}{|c|c|c|c|c|c|c|c|c|c|c|c|c|}
\hline \multirow[t]{2}{*}{ Study } & \multirow[t]{2}{*}{ Specimen } & \multirow[t]{2}{*}{ Side } & \multicolumn{3}{|c|}{$\begin{array}{l}\text { Vertical diameter of IOF } \\
\text { (VD-IOF) [mm] }\end{array}$} & \multicolumn{3}{|c|}{$\begin{array}{l}\text { Transvers diameter of IOF } \\
\text { (TD-IOF) [mm] }\end{array}$} & \multicolumn{3}{|c|}{ Average diameter of IOF } & \multirow{2}{*}{$\begin{array}{l}\text { The largest di- } \\
\text { ameter of IOF } \\
\text { Total }\end{array}$} \\
\hline & & & $F$ & $M$ & Total & $F$ & M & Total & $\mathbf{F}$ & $M$ & Total & \\
\hline \multirow{2}{*}{$\begin{array}{l}\text { Kara et al. } \\
{[36]}\end{array}$} & \multirow[t]{2}{*}{ CT } & $\mathrm{R}$ & & & & & & & \multirow{2}{*}{$\begin{array}{l}1.51 \pm \\
0.49^{*}\end{array}$} & \multirow{2}{*}{\multicolumn{2}{|c|}{$\begin{array}{c}2.13 \pm \\
0.75^{*}\end{array}$}} & \\
\hline & & $\mathrm{L}$ & & & & & & & & & & \\
\hline \multirow{2}{*}{$\begin{array}{l}\text { Berge and } \\
\text { Bergman [10] }\end{array}$} & \multirow[t]{2}{*}{ Dry skull } & $\mathrm{R}$ & & & & & & & \multirow{2}{*}{\multicolumn{3}{|c|}{$3.42 \times 2.40$}} & \\
\hline & & L & & & & & & & & & & \\
\hline \multirow[t]{2}{*}{ Lang [40] } & \multirow[t]{2}{*}{ Dry skull } & $\mathrm{R}$ & & & & & & & & & & $4.57(7.9-2.5)$ \\
\hline & & $\mathrm{L}$ & & & & & & & & & & $4.56(7.5-2.6)$ \\
\hline \multirow{2}{*}{$\begin{array}{l}\text { Song et al. } \\
{[69]}\end{array}$} & \multirow[t]{2}{*}{ Cadaver } & $R$ & & & & & & & & & & \multirow{2}{*}{$5 \pm 1$} \\
\hline & & $\mathrm{L}$ & & & & & & & & & & \\
\hline \multirow[t]{2}{*}{ Tezer [73] } & \multirow[t]{2}{*}{ Dry skull } & $\mathrm{R}$ & & & & & & & & & & $4.10 \pm 0.87$ \\
\hline & & $\mathrm{L}$ & & & & & & & & & & $4.33 \pm 0.94$ \\
\hline \multirow[t]{2}{*}{ Taşpinar [72] } & \multirow[t]{2}{*}{ MDBT } & $\mathrm{R}$ & $1.70=$ & $=0.43$ & $1.71 \pm$ & $1.89 \pm$ & $=0.44$ & & & & & \\
\hline & & $L$ & 1.72 & $=0.40$ & 0.42 & $1.88 \pm$ & 0.44 & $1.88 \pm 0.44$ & & & & \\
\hline Chung et al. & Dry skull & $\mathrm{R}$ & & & & $4.5 \pm 1.1$ & $4.9 \pm 1.5$ & & & & & \\
\hline & & $\mathrm{L}$ & & & & $5.0 \pm 1.4$ & $4.9 \pm 1.5$ & & & & & \\
\hline Apinhasmit et al. & Dry skull & $\mathrm{R}$ & & & & & & $325+062$ & & & & \\
\hline & & $\mathrm{L}$ & & & & & & $3.35 \pm 0.62$ & & & & \\
\hline Boopathi et al. & Dry skull & $R$ & $2.79=$ & $=0.79$ & $2.82 \pm$ & $2.73 \pm$ & 0.73 & & & & & \\
\hline & & $\mathrm{L}$ & 2.85 & $=0.80$ & 0.79 & $3.00 \pm$ & $=0.81$ & $2.87 \pm 0.18$ & & & & \\
\hline Aziz et al. & Cadaver & $\mathrm{R}$ & & & & $4.4 \pm 1.0$ & $4.8 \pm 1.0$ & & & & & \\
\hline [6] & & $\mathrm{L}$ & & & & $4.5 \pm 1.0$ & $4.6 \pm 1.2$ & & & & & \\
\hline Cisneiros de & Dry skull & $\mathrm{R}$ & 4.0 & 4.5 & 4.0 & 4.0 & 4.0 & $4.0(3.5-4.5)$ & & & & \\
\hline Oliveira et al. & & & $(4.0-5.0)$ & $(4.0-5.0)$ & $(4.0-5.0)^{*}$ & $(3.0-4.0)^{*}$ & $(3.5-4.5)^{*}$ & & & & & \\
\hline & & L & 4.0 & 4.0 & 4.0 & 3.5 & 4.0 & $4.0(3.5-4.5)$ & & & & \\
\hline & & & $(3.5-$ & $14.0-$ & (3.5- & (3.0- & (3.5- & & & & & \\
\hline & & & $4.12)^{*}$ & $5.0)^{*}$ & $4.5)^{*}$ & $4.0)^{*}$ & $4.5)^{*}$ & & & & & \\
\hline Takahashi et al. & Cadaver & $\mathrm{R}$ & & & & 510 & 570 & 550 & & & & \\
\hline [71] & & $\mathrm{L}$ & & & & 5.10 & 5.10 & 5.50 & & & & \\
\hline Chrcanovic et al. & Dry skull & $\mathrm{R}$ & & & & $3.20 \pm$ & $3.31 \pm$ & & & & & \\
\hline & & L & & & & 0.81 & 0.81 & $3.23 \pm 0.81$ & & & & \\
\hline Singh [68] & Dry skull & $\mathrm{R}$ & $3.39=$ & $=0.96$ & $3.57 \pm$ & $3.19 \pm$ & 1.18 & $325+12$ & & & & \\
\hline & & $\mathrm{L}$ & $3.75=$ & $=1.07$ & 1.0 & $3.52 \pm$ & 1.35 & $3.35 \pm 1.3$ & & & & \\
\hline Aggarwal et al. & Dry skull & $\mathrm{R}$ & $3.50=$ & $=1.16$ & $3.54 \pm$ & $2.65 \pm$ & $=0.93$ & & & & & \\
\hline & & L & $3.58=$ & $=1.07$ & 1.11 & $2.80 \pm$ & 0.98 & \pm 0.95 & & & & \\
\hline $\begin{array}{l}\text { Sinanoğlu et al. } \\
\text { [67] }\end{array}$ & СВCT & $\mathrm{R}$ & $\begin{array}{c}1.85 \pm \\
0.2\end{array}$ & $\begin{array}{c}2.83 \pm \\
0.1\end{array}$ & & $\begin{array}{c}3.82 \pm \\
0.2\end{array}$ & $\begin{array}{c}4.83 \pm \\
0.1\end{array}$ & & & & & \\
\hline & & $\mathrm{L}$ & $\begin{array}{c}1.83 \pm \\
0.2\end{array}$ & $\begin{array}{c}2.63 \pm \\
0.2\end{array}$ & & $\begin{array}{c}3.97 \pm \\
0.2\end{array}$ & $\begin{array}{c}4.74 \pm \\
0.3\end{array}$ & & & & & \\
\hline Present study & СВCT & $\mathbf{R}$ & $\begin{array}{c}3.21 \pm \\
0.45\end{array}$ & $\begin{array}{c}3.77 \pm \\
1.30^{*}\end{array}$ & $\begin{array}{c}3.49 \pm \\
1.01^{*}\end{array}$ & $\begin{array}{l}3.32 \pm \\
0.47^{*}\end{array}$ & $\begin{array}{c}3.42 \pm \\
0.56\end{array}$ & $3.37 \pm 0.52^{*}$ & & & & \\
\hline & & L & $\begin{array}{c}3.11 \pm \\
0.55\end{array}$ & $\begin{array}{l}3.46 \pm \\
0.80^{*}\end{array}$ & $\begin{array}{l}3.29 \pm \\
0.71^{*}\end{array}$ & $\begin{array}{l}3.15 \pm \\
0.45^{*}\end{array}$ & $\begin{array}{c}3.29 \pm \\
0.66\end{array}$ & $3.22 \pm 0.57^{*}$ & & & & \\
\hline
\end{tabular}

${ }^{*}$ Significant difference; L — left; R — right; F — female; M — male; CBCT — cone-beam computed tomography; CT — computed tomography; VD-IOF — vertical diameter of infraorbital foramen; TD-IOF — transvers diameter of infraorbital foramen

and Mailleux et al. [47] reported cases of IOC passing through in the maxillary sinus. Ference et al. [26] reported this variation in $12.5 \%$ of 200 cases, Lantos et al. [41] reported it in $10.8 \%$ of 500 cases and Yenigun et al. [79] reported in $12.3 \%$ of 750 cases. In the present study, this variation was found to be $7 \%$ of
150 cases (Fig. 2). Functional endoscopic sinus surgery (FESS) is the standard surgical treatment for chronic inflammatory and allergic synonasal disease [23]. FESS procedure is based on the theory that the opening of the obstructed pathway of osteomeatal drainage complexes will restore the normal ventilation of the sinuses 
Table 5. Comparison of IOF-IOM and IOF-ML with the literature

\begin{tabular}{|c|c|c|c|c|c|c|c|c|}
\hline \multirow{2}{*}{ Study } & \multirow[t]{2}{*}{ Specimen } & \multirow[t]{2}{*}{ Side } & \multicolumn{3}{|c|}{ IOF-IOM [mm] } & \multicolumn{3}{|c|}{ IOF-ML [mm] } \\
\hline & & & $\mathbf{F}$ & M & Total & $\mathbf{F}$ & M & Total \\
\hline & \multirow[t]{2}{*}{ Dry skull } & $\mathrm{R}$ & \multirow{2}{*}{\multicolumn{2}{|c|}{$\begin{array}{l}6.37 \pm 1.52 \\
6.28 \pm 1.25\end{array}$}} & \multirow[b]{2}{*}{$6.33 \pm 1.39$} & \multirow{2}{*}{\multicolumn{2}{|c|}{$\begin{array}{l}25.63 \pm 2.27 \\
25.74+2.50\end{array}$}} & \multirow[b]{2}{*}{$25.69 \pm 2.37$} \\
\hline & & $\mathrm{L}$ & & & & & & \\
\hline Kazkayasi et al. [38] & Dry skull & $\mathrm{R}$ & & & & & & \\
\hline & & $\mathrm{L}$ & & & $1.19 \pm 1.39$ & & & \\
\hline Kazkayasi et al. [38] & X-ray & $\mathrm{R}$ & & & $745+0.95$ & & & \\
\hline & & $\mathrm{L}$ & & & $1.4 \mathrm{~J}-0.90$ & & & \\
\hline Hwang et al. [34] & HRCT & $\mathrm{R}$ & $9.4 \pm 1.6$ & $9.7 \pm 1.7$ & $9.6 \pm 1.7$ & $29.1 \pm 1.8$ & $26.9 \pm 1.9$ & $26.5 \pm 1.9$ \\
\hline & & $\mathrm{L}$ & & 年 & $3.0-1.1$ & $20.1-1.0$ & & $20.5-1.5$ \\
\hline Cutright et al. [21] & Cadaver (white) & $\mathrm{R}$ & $5.8 \pm 0.3$ & $7.1 \pm 0.3$ & & $24.5 \pm 0.3^{*}$ & $27.4 \pm 0.5$ & \\
\hline & & $\mathrm{L}$ & $0.0-0.0$ & . & & - & - & \\
\hline Cutright et al. [21] & Cadaver (black) & $\mathrm{R}$ & $5.7 \pm 0.2$ & $6.9 \pm 0.3$ & & $26.4 \pm 0.4^{*}$ & $29.5 \pm 0.5$ & \\
\hline Raschke et al. [61] & Spiral CT & $\frac{L}{R}$ & & & & & & \\
\hline & & $\mathrm{L}$ & $8.25 \pm 0.54$ & $8.61 \pm 0.64$ & & & & \\
\hline Ercikti et al. [25] & Cadaver & $\mathrm{R}$ & $7.8 \pm 1.0$ & $9.6 \pm 1.2$ & $88+10$ & $27.5 \pm 2.7$ & $32.0 \pm 2.0$ & $303+27$ \\
\hline & & $\mathrm{L}$ & $7.9 \pm 0.8$ & $9.8 \pm 0.9$ & $8.8 \pm 1.0$ & $31.2 \pm 3.1$ & $30.5 \pm 2.9$ & $30.3 \pm 2.1$ \\
\hline Lokanayaki [25] & Dry skull & $\mathrm{R}$ & & & $6.12 \pm 1.43$ & & & \\
\hline & & $\mathrm{L}$ & & & $6.53 \pm 1.53$ & & & \\
\hline Saylam et al. [66] & Cadaver and & $\mathrm{R}$ & 83 & 109 & & & & \\
\hline & dry skull & $\mathrm{L}$ & 8,3 & 10,9 & & & & \\
\hline Rahman et al. [59] & Cadaver & $\mathrm{R}$ & & & 8 & & & 26 \\
\hline & & $\mathrm{L}$ & & & 0 & & & $<0$ \\
\hline Aziz et al. [6] & Cadaver & $\mathrm{R}$ & $8.1 \pm 1.6$ & $8.5 \pm 2.1$ & & $25.5 \pm 3.6$ & $27.9 \pm 4.9$ & \\
\hline & & $\mathrm{L}$ & $7.6 \pm 1.6$ & $8.5 \pm 2.3$ & & $26.9 \pm 2.7$ & $27.5 \pm 3.7$ & \\
\hline Apinhasmit et al. [5] & Dry skull & $\mathrm{R}$ & $871+151 *$ & $953+223 *$ & & $27.29+212 *$ & $2910+213 *$ & \\
\hline & & $\mathrm{L}$ & $0.71-1.01$ & $9.00-2.20$ & $9.20 \pm-2.00$ & $21 . \angle 9 \pm 2.12$ & $29.10 \pm-2.10$ & $20.40 \perp-2.29$ \\
\hline Boopathi et al. [12] & Dry skull & $\mathrm{R}$ & 6.49 & $=1.26$ & & & & \\
\hline & & $\mathrm{L}$ & 6.65 & $=1.30$ & $6.5 / \pm 1.28$ & & & \\
\hline Cisneiros de Oliveira & Dry skull & $\mathrm{R}$ & $8.0(7.0-9.0)$ & $8.0(7.0-9.0)$ & & & & \\
\hline et al. [20] & & $\mathrm{L}$ & $8.0(7.0-9.0)$ & $8.0(6.5-9.0)$ & & & & \\
\hline Chrcanovic et al. [18] & Dry skull & $\mathrm{R}$ & $6.35 \pm 1.67$ & $6.63 \pm 1.75$ & $6.41 \pm 1.69$ & $24.67 \pm 2.41$ & $26.48 \pm 2.58$ & $25.26 \pm 2.60$ \\
\hline & & $\mathrm{L}$ & & & & & & \\
\hline Singh [68] & Dry skull & $\mathrm{R}$ & 6.12 & 1.79 & $6.16 \pm 1.8$ & & & \\
\hline & & $\mathrm{L}$ & 6.19 & $=1.81$ & $0.10 \pm 1.0$ & & & \\
\hline Taşpınar [72] & MDCT & $\mathrm{R}$ & 7.44 & 1.34 & & $27.16=$ & $\pm 3.80^{*}$ & \\
\hline & & $\mathrm{L}$ & 7.55 & 1.37 & $1.50 \pm 1.36$ & $26.47=$ & $\pm 2.40^{*}$ & $2 b .81 \pm 3.19$ \\
\hline Tezer [73] & Dry skull & $\mathrm{R}$ & 6.98 & $=1.68$ & & 28.27 & \pm 2.38 & \\
\hline & & $\mathrm{L}$ & 6.98 & $=1.75$ & & 28.67 & \pm 2.59 & \\
\hline Karakas et al. [37] & Dry skull & $\mathrm{R}$ & & & $67+19$ & & & \\
\hline & & $L$ & & & $0.1 \pm 1.9$ & & & \\
\hline Elias et al. [22] & Dry skull & $\mathrm{R}$ & $6.71=$ & $1.70^{*}$ & & & & \\
\hline & & $\mathrm{L}$ & $6.83=$ & $1.83^{*}$ & & & & \\
\hline Agthong et al. [3] & Dry skull & $\mathrm{R}$ & $7.5 \pm 0.3$ & $8.0 \pm 0.3$ & $7.8 \pm 0.2$ & & & \\
\hline & & $\mathrm{L}$ & $7.8 \pm 0.2$ & $8.2 \pm 0.3$ & $8.0 \pm 0.2$ & & & \\
\hline Gupta [29] & Dry skull & $\mathrm{R}$ & & 1.6 & $70+16$ & 28.0 & \pm 2.8 & 26 \\
\hline & & $\mathrm{L}$ & 7.0 & $=1.7$ & $0 \pm 1.0$ & & \pm 2.5 & $20.5 \pm 2.0$ \\
\hline Macedo et al. [46] & Dry skull & $\mathrm{R}$ & $6.28=$ & $1.79^{*}$ & $637+169$ & & & \\
\hline & & $\mathrm{L}$ & 6.45 & $1.76^{*}$ & $0.31 \pm 1.09$ & & & \\
\hline Ukoha et al. [75] & Dry skull & $\mathrm{R}$ & 6.94 & $=2.57$ & $738-2$ & & & \\
\hline & & $\mathrm{L}$ & 7.83 & $=1.86$ & $1.38 \pm 2.28$ & & & \\
\hline Gour et al. [28] & Dry skull & $\mathrm{R}$ & $6.20 \pm 1.859$ & $6.55 \pm 1.667$ & $6.50 \pm 1.744$ & & & \\
\hline & & $\mathrm{L}$ & & & & & & \\
\hline Xu et al. [78] & CT & $\mathrm{R}$ & $9.23 \pm 1.90$ & $9.30 \pm 1.55$ & $9.26 \pm 1.68$ & $26.03 \pm 1.94$ & $27.21 \pm 2.25$ & $26.75 \pm 2.20$ \\
\hline & & $\mathrm{L}$ & $8.81 \pm 1.76$ & $9.21 \pm 1.33$ & $9.04 \pm 1.52$ & $26.19 \pm 1.84$ & $26.97 \pm 2.28$ & $26.69 \pm 2.15$ \\
\hline Robinson and Wormald [62] & Cadaver & $\mathrm{R}$ & & & & & & \\
\hline & & $\mathrm{L}$ & & & 6,8 & & & \\
\hline Abed et al. [1] & Cadaver & $\mathrm{R}$ & & & $895+153$ & & & \\
\hline & & $\mathrm{L}$ & & & $8.95 \pm 1.53$ & & & \\
\hline Lee et al. [42] & CT & $\mathrm{R}$ & $8.33 \pm 1.50$ & $8.49 \pm 1.50$ & 8.42 & & & \\
\hline & & $\mathrm{L}$ & $8.34 \pm 1.80$ & $8.50 \pm 1.60$ & 8.42 & & & \\
\hline Michalek et al. [50] & Cadaver & $\mathrm{R}$ & & & $760+130$ & & & \\
\hline & (ultrasound) & $\mathrm{L}$ & & & $1.60 \pm 1.30$ & & & \\
\hline Michalek et al. [50] & Cadaver (Direk & $\mathrm{R}$ & & & & & & \\
\hline & measurement) & $\mathrm{L}$ & & & $6.10 \pm 0.90$ & & & \\
\hline Present study & СВCT & $\mathbf{R}$ & $7.15 \pm 1.22$ & $7.79 \pm 1.49$ & $7.47 \pm 1.40$ & $22.65 \pm 2.17$ & $24.39 \pm 2.00$ & $23.52 \pm 2.26$ \\
\hline & & $\mathbf{L}$ & $7.10 \pm 1.30$ & $7.67 \pm 1.47$ & $7.39 \pm 1.41$ & $22.67 \pm 2.39$ & $24.19 \pm 2.15$ & $23.43 \pm 2.39$ \\
\hline
\end{tabular}

*Significant difference; L — left; R — right; F — female; $\mathrm{M}$ — male; CBCT — cone-beam computed tomography; CT — computed tomography; HRCT — high resolution computed tomography; MDCT — multiple detector computed tomography; IOF-IOM — the distance between IOF and infraorbital margin; IOF-ML — the distance between IOF and the mid-sagittal plane 
Table 6. Comparison of IOF-PM, IOF-LOR, IOF-FZS and ST with the literature

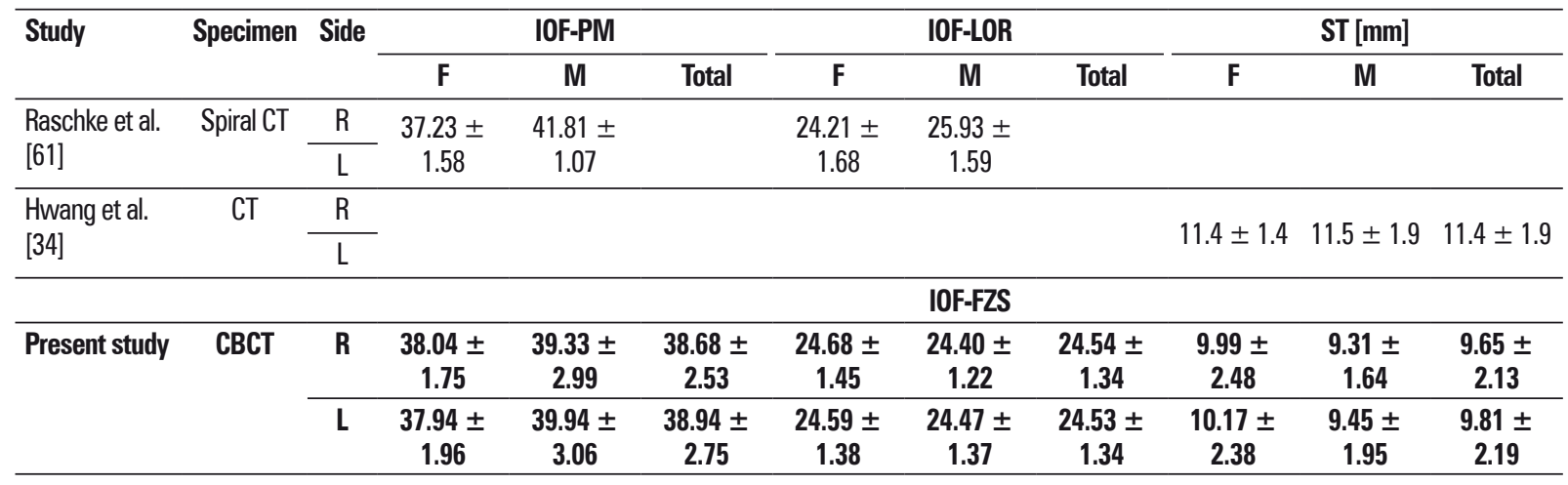

$\mathrm{L}$ - left; $\mathrm{R}$ - right; F — female; $\mathrm{M}$ - male; CBCT — cone-beam computed tomography; CT — computed tomography; IOF-PM — the distance between IOF and the occlusal plane of the second premolar tooth; IOF-LOR — the distance between IOF and lateral orbital rim; IOF-FZS — the distance between IOF and vertical axis of lateral rim of frontozygomatic suture;

ST — skin thickness over IOF (skin thickness)

Table 7. Comparison of IOF-LNW and IOF-PA with the literature

\begin{tabular}{|c|c|c|c|c|c|c|c|c|}
\hline \multirow[t]{2}{*}{ Study } & \multirow[t]{2}{*}{ Specimen } & \multirow[t]{2}{*}{ Side } & \multicolumn{3}{|c|}{ IOF-LNW [mm] } & \multicolumn{3}{|c|}{ IOF-PA [mm] } \\
\hline & & & $\mathbf{F}$ & M & Total & $\mathbf{F}$ & M & Total \\
\hline \multirow[t]{2}{*}{ Aggarwal et al. [2] } & \multirow[t]{2}{*}{ Dry skull } & $\mathrm{R}$ & & & & \multicolumn{2}{|c|}{$15.51 \pm 1.63$} & \multirow{2}{*}{$15.19 \pm 1.70$} \\
\hline & & L & & & & 14.87 & 1.73 & \\
\hline \multirow[t]{2}{*}{ Kazkayasi et al. [38] } & \multirow[t]{2}{*}{ Dry skull } & $\mathrm{R}$ & & & & & & \multirow{2}{*}{$17.23 \pm 2.64$} \\
\hline & & $\mathrm{L}$ & & & & & & \\
\hline \multirow[t]{2}{*}{ Kazkayasi et al. [38] } & \multirow[t]{2}{*}{ X-ray } & $\mathrm{R}$ & & & \multirow{2}{*}{$14.31 \pm 1.96$} & & & \\
\hline & & L & & & & & & \\
\hline \multirow[t]{2}{*}{ Raschke et al. [61] } & \multirow[t]{2}{*}{ Spiral CT } & $\mathrm{R}$ & & & & \multirow{2}{*}{\multicolumn{2}{|c|}{$15.69 \pm 0.76 \quad 17.43 \pm 1.19$}} & \\
\hline & & $L$ & & & & & & \\
\hline \multirow[t]{2}{*}{ Lokanayaki [45] } & \multirow[t]{2}{*}{ Dry skull } & $\mathrm{R}$ & & & & & & $16.58 \pm 2.37$ \\
\hline & & L & & & & & & $16.38 \pm 2.25$ \\
\hline \multirow[t]{2}{*}{ Rahman et al. [59] } & \multirow[t]{2}{*}{ Cadaver } & $\mathrm{R}$ & & & & & & \multirow{2}{*}{17} \\
\hline & & L & & & & & & \\
\hline \multirow[t]{2}{*}{ Chrcanovic et al. [18] } & \multirow[t]{2}{*}{ Dry skull } & $\mathrm{R}$ & & & & \multirow{2}{*}{$14.37 \pm 2.04$} & \multirow{2}{*}{$15.44 \pm 1.79$} & \multirow{2}{*}{$14.72 \pm 2.02$} \\
\hline & & $\mathrm{L}$ & & & & & & \\
\hline \multirow[t]{2}{*}{ Singh [68] } & \multirow[t]{2}{*}{ Dry skull } & $\mathrm{R}$ & & & & \multicolumn{2}{|c|}{$15.31 \pm 1.77$} & \\
\hline & & L & & & & 15.80 & 2.86 & 10.00 \\
\hline Taşpınar [72] & MDCT & $\mathrm{R}$ & 16.58 & $=1.85$ & $1663+181$ & & & \\
\hline & & L & 16.68 & $=1.77$ & $10.00-1.01$ & & & \\
\hline Elias et al. [22] & Dry skull & $\mathrm{R}$ & & & & 13.28 & $2.17^{*}$ & \\
\hline & & $\mathrm{L}$ & & & & 13.31 & $2.19^{*}$ & \\
\hline Macedo et al. [46] & Dry skull & $\mathrm{R}$ & & & & 17.75 & \pm 2.10 & 195 \\
\hline & & L & & & & 17.60 & 2.04 & $17.08-1.50$ \\
\hline Ukoha et al. [75] & Dry skull & $\mathrm{R}$ & & & & 19.36 & \pm 3.54 & 100 \\
\hline & & $\mathrm{L}$ & & & & 18.27 & 2.94 & $10.02-0.20$ \\
\hline Present study & СВСТ & $\mathbf{R}$ & $9.24 \pm 2.34$ & $9.89 \pm 2.42$ & $9.57 \pm 2.39$ & & & \\
\hline & & $\mathbf{L}$ & $8.77 \pm 2.19$ & $9.90 \pm 2.41$ & $9.34 \pm 2.36$ & & & \\
\hline
\end{tabular}

*Significant difference; L — left; $\mathrm{R}$ — right; F — female; $\mathrm{M}$ — male; CBCT — cone-beam computed tomography; MDCT — multidetector computed tomography; IOF-LNW — the distance between infraorbital foramen and the lateral wall of the nasal cavity; IOF-PA — the distance between infraorbital foramen and preform aperture 
in cases of chronic sinusitis [17]. Prior to FESS, Mailleux et al. [47] and Elnil et al. [23] proposed CT imaging as a preoperative procedure to reduce complications. Lantos et al. [41] reported a high risk of ION injury in maxillary sinus with chronic inflammation, neoplasms, and patients with these variations in resection of an inverted papilloma require an antral punch to introduce a balloon dilation catheter to approach the maxillary infundibulum. A window is opened through the gingivobuccal sulcus in the anterior wall of the maxilla to reach maxillary sinus in Caldwell Luc operation, which is one of the preferred methods in various situations such as chronic rhinosinusitis, paranasal papilloma and foreign body removal $[8,9,11,56]$. For these reasons, the variations of $I O C$ in the maxillary sinus should be considered in the surgical procedures. Although for the cases that IOC in maxillary sinus is less likely to have infraorbital neurovascular bundle injury in orbital floor fractures as they move away from the orbital floor.

Leo et al. [44] identified two separate $I O C$ with ION that opened to the anterior surface of maxilla in a cadaver. Von Arx et al. [76] defined canalis sinuosus, as a tortuous bone channel originating from the IOC slightly posterior to the IOF and coursing in an anteromedial direction to the anterior wall of the nasal antrum below the orbital margin. Rusu et al. [64] identified a case in which the IOC was absent and the lateroantral duct was present. In this study a very rare case was detected; $I O C$ was divided into two canals in the maxillary sinus and the smaller one was directed to the lateral wall of the nasal cavity and opened to the inferior nasal meatus in front of the opening of the nasolacrimal duct (Fig. 3).

\section{CONCLUSIONS}

In conclusion, we suggest that the parameters found in the present study may facilitate prediction of the location of the ION. Knowledge of this exact position in relation to easily measurable parameters may decrease the risk of ION injury during surgical approaches directed to this region and might serve as a guide during local anaesthetic interventions for dentistry, ophthalmology, plastic surgery, rhinology, neurosurgery and dermatology.

\section{Ethical standards}

This study was approved by the ethics committee of Gaziantep University (approval date and number: 07 March 2016; 2016/72). We declare that this human study has been approved by the ethics commit- tee of Gaziantep University and has, therefore, been performed in accordance with the ethical standards laid down in the 1964 Declaration of Helsinki and its later amendments.

\section{Acknowledgements}

The authors are greatful to Associate Professor Dr. Seval KUL, Department of Biostatistics, School of Medicine, Gaziantep University for the support.

\section{REFERENCES}

1. Abed SF, Shams PN, Shen S, et al. Morphometric and geometric anatomy of the caucasian orbital floor. Orbit. 2011; 30(5): 214-220, doi: 10.3109/01676830.2010.539768, indexed in Pubmed: 21812531.

2. Aggarwal A, Kaur H, Gupta T, et al. Anatomical study of the infraorbital foramen: A basis for successful infraorbital nerve block. Clin Anat. 2015; 28(6): 753-760, doi: 10.1002/ ca.22558, indexed in Pubmed: 26119635.

3. Agthong S, Huanmanop T, Chentanez V. Anatomical variations of the supraorbital, infraorbital, and mental foramina related to gender and side. J Oral Maxillofac Surg. 2005; 63(6): 800-804, doi: 10.1016/j.joms.2005.02.016, indexed in Pubmed: 15944977.

4. Aitasalo K, Kinnunen I, Palmgren J, et al. Repair of orbital floor fractures with bioactive glass implants. J Oral Maxillofac Surg. 2001; 59(12): 1390-5; discussion 1395, doi: 10.1053/ joms.2001.27524, indexed in Pubmed: 11732018.

5. Apinhasmit W, Chompoopong S, Methathrathip D, et al. Supraorbital Notch/Foramen, Infraorbital Foramen and Mental Foramen in Thais: anthropometric measurements and surgical relevance. J Med Assoc Thai. 2006; 89(5): 675-682, indexed in Pubmed: 16756055.

6. Aziz SR, Marchena JM, Puran A. Anatomic characteristics of the infraorbital foramen: a cadaver study. J Oral Maxillofac Surg. 2000; 58(9): 992-996, doi: 10.1053/joms.2000.8742, indexed in Pubmed: 10981979.

7. Bahşi I, Orhan M, Kervancıoğlu P, et al. Anatomical evaluation of nasopalatine canal on cone beam computed tomography images. Folia Morphol. 2019; 78(1): 153-162, doi: 10.5603/ FM.a2018.0062, indexed in Pubmed: 30009362.

8. Balıkçı HH, Özbay I, Karakaş M. Etmoid sinüste saçma tanesi: olgu sunumu. ODÜ Tıp Dergisi. 2015; 2(3): 126-129.

9. Becker SS, Roberts DM, Beddow PA, et al. Comparison of maxillary sinus specimens removed during Caldwell-Luc procedures and traditional maxillary sinus antrostomies. Ear Nose Throat J. 2011; 90(6): 262-266, doi: 10.1177/0145561 31109000607, indexed in Pubmed: 21674469.

10. Berge JK, Bergman RA. Variations in size and in symmetry of foramina of the human skull. Clin Anat. 2001; 14(6): 406-413, doi: 10.1002/ca.1075, indexed in Pubmed: 11754234.

11. Berkiten G, Bal M, Kumral TL, et al. Schneiderian papilloma: our experience over 12 years and literature review. Nobel Medicus. 2015; 11(2): 19-23.

12. Boopathi S, Chakravarthy Marx S, Dhalapathy SL, et al. Anthropometric analysis of the infraorbital foramen in a South Indian population. Singapore Med J. 2010; 51(9): 730-735, indexed in Pubmed: 20938615.

13. Bösenberg AT, Kimble FW. Infraorbital nerve block in neonates for cleft lip repair: anatomical study and clinical application. Br J Anaesth. 1995; 74(5): 506-508, indexed in Pubmed: 7772421.

14. Brandão FH, Machado M, Aquino J, et al. The foramen and infraorbital nerve relating to the surgery for external access to the maxillary sinus (Caldwell-Luc). Int Arch Otorhinolarnygol. 2008; 12(3): 342-346. 
15. Chan BJ, Koushan K, Liszauer A, et al. latrogenic globe penetration in a case of infraorbital nerve block. Can J Ophthalmol. 2011; 46(3): 290-291, doi: 10.1016/j.jcjo.2011.05.012, indexed in Pubmed: 21784222.

16. Chandra RK, Kennedy DW. Surgical implications of an unusual anomaly of the infraorbital nerve. Ear Nose Throat J. 2004; 83(11): 766-767, indexed in Pubmed: 15628633.

17. Chong VF, Fan YF, Lau D, et al. Functional endoscopic sinus surgery (FESS): what radiologists need to know. Clin Radiol. 1998; 53(9): 650-658, indexed in Pubmed: 9766718.

18. Chrcanovic BR, Abreu MH, Custódio AL. A morphometric analysis of supraorbital and infraorbital foramina relative to surgical landmarks. Surg Radiol Anat. 2011; 33(4): 329-335, doi: 10.1007/s00276-010-0698-1, indexed in Pubmed: 20625730.

19. Chung MS, Kim HJ, Kang HS, et al. Locational relationship of the supraorbital notch or foramen and infraorbital and mental foramina in Koreans. Acta Anat (Basel). 1995; 154(2): 162-166, indexed in Pubmed: 8722516.

20. Cisneiros de Oliveira LC, Silveira MP, de Almeida Júnior E, et al. Morphometric study on the infraorbital foramen in relation to sex and side of the cranium in northeastern Brazil. Anat Cell Biol. 2016; 49(1): 73-77, doi: 10.5115/acb.2016.49.1.73, indexed in Pubmed: 27051570.

21. Cutright B, Quillopa N, Schubert W. An anthropometric analysis of the key foramina for maxillofacial surgery. J Oral Maxillofac Surg. 2003; 61(3): 354-357, doi: 10.1053/ joms.2003.50070, indexed in Pubmed: 12618976.

22. Elias MG, Silva RB, Pimentel ML, et al. Morphometric analysis of the infraorbital foramen and acessories foraminas in brazilian skulls. Int J Morphol. 2004; 22(4), doi: 10.4067/ s0717-95022004000400006.

23. Elnil H, Al-Tubaikh JA, El Beltagi AH. Into the septum I go, a case of bilateral ectopic infraorbital nerves: a not-to-miss preoperative sinonasal CT variant. Neuroradiol J. 2014; 27(2): 146-149, doi: 10.15274/NRJ-2014-10033, indexed in Pubmed: 24750699.

24. Eppley B. Re: cutaneous distribution of infraorbital nerve. J Craniofac Surg. 2004; 15(1): 5, doi: 10.1097/00001665200401000-00003.

25. Ercikti N, Apaydin N, Kirici Y. Location of the infraorbital foramen with reference to soft tissue landmarks. Surg Radiol Anat. 2017; 39(1): 11-15, doi: 10.1007/s00276-016-1683-0, indexed in Pubmed: 27146295.

26. Ference EH, Smith SS, Conley D, et al. Surgical anatomy and variations of the infraorbital nerve. Laryngoscope. 2015; 125(6): 1296-1300, doi: 10.1002/lary.25089, indexed in Pubmed: 25992806.

27. Feriani G, Hatanaka E, Torloni MR, et al. Infraorbital nerve block for postoperative pain following cleft lip repair in children. Cochrane Database Syst Rev. 2016; 4: CD011131, doi: 10.1002/14651858.CD011131.pub2, indexed in Pubmed: 27074283.

28. Gour K, Nair S, Trivedi G, et al. Anthropometric measurements of infraorbital foramen in dried human skulls. Int J Biol Med Res. 2012; 3(3): 2003-2006.

29. Gupta T. Localization of important facial foramina encountered in maxillo-facial surgery. Clin Anat. 2008; 21(7): 633-640, doi: 10.1002/ca.20688, indexed in Pubmed: 18773483.

30. Harris D, Buser D, Dula K, et al. E.A.O. Guidelines for the use of Diagnostic Imaging in Implant Dentistry. A consensus workshop organized by the European Association for Osseointegration in Trinity College Dublin. Clin Oral Impl Res. 2002; 13(5): 566-570, doi: 10.1034/j.1600-0501.2002.130518.x.

31. Higashizawa T, Koga Y. Effect of infraorbital nerve block under general anesthesia on consumption of isoflurane and postoperative pain in endoscopic endonasal maxillary sinus surgery. J Anesth. 2001; 15(3): 136-138, doi: 10.1007/ s005400170014, indexed in Pubmed: 14566510.

32. Huanmanop T, Agthong S, Chentanez V. Surgical anatomy of fissures and foramina in the orbits of Thai adults. J Med
Assoc Thai. 2007; 90(11): 2383-2391, indexed in Pubmed: 18181324

33. Hwang K, Suh MS, Chung InH. Cutaneous distribution of infraorbital nerve. J Craniofac Surg. 2004; 15(1): 3-5; discussion 5, indexed in Pubmed: 14704552.

34. Hwang SeH, Kim SW, Park CS, et al. Morphometric analysis of the infraorbital groove, canal, and foramen on threedimensional reconstruction of computed tomography scans. Surg Radiol Anat. 2013; 35(7): 565-571, doi: 10.1007/ s00276-013-1077-5, indexed in Pubmed: 23404562.

35. Jonnavithula N, Durga P, Kulkarni DK, et al. Bilateral intraoral, infra-orbital nerve block for postoperative analgesia following cleft lip repair in paediatric patients: comparison of bupivacaine vs bupivacaine-pethidine combination. Anaesthesia. 2007; 62(6): 581-585, doi: 10.1111/j.13652044.2007.05028.x, indexed in Pubmed: 17506736.

36. Kara SA, Ünal B, Erdal H, et al. İnfraorbital Foramen Anatomisinin Radyolojik Analizi. KBB ve BBC Dergisi. 2003; 11(1): 17-21.

37. Karakaș P, Bozkir MG, Oguz O. Morphometric measurements from various reference points in the orbit of male Caucasians. Surg Radiol Anat. 2003; 24(6): 358-362, doi: 10.1007/ s00276-002-0071-0, indexed in Pubmed: 12652362.

38. Kazkayasi M, Ergin A, Ersoy M, et al. Certain anatomical relations and the precise morphometry of the infraorbital foramen--canal and groove: an anatomical and cephalometric study. Laryngoscope. 2001; 111(4 Pt 1): 609-614, doi: 10.1097/00005537200104000-00010, indexed in Pubmed: 11359128.

39. Kazkayasi M, Ergin A, Ersoy M, et al. Microscopic anatomy of the infraorbital canal, nerve, and foramen. Otolaryngol Head Neck Surg. 2003; 129(6): 692-697, doi: 10.1016/S019459980301575-4, indexed in Pubmed: 14663437.

40. Lang J. Clinical Anatomy of the Head: Neurocranium - Orbit. Craniocervical Regions. Berlin: Springer Science \& Business Media. 2012.

41. Lantos JE, Pearlman AN, Gupta A, et al. Protrusion of the Infraorbital Nerve into the Maxillary Sinus on CT: Prevalence, Proposed Grading Method, and Suggested Clinical Implications. AJNR Am J Neuroradiol. 2016; 37(2): 349-353, doi: 10.3174/ajnr.A4588, indexed in Pubmed: 26564432.

42. Lee T, Lee H, Baek S. A three-dimensional computed tomographic measurement of the location of infraorbital foramen in East Asians. J Craniofac Surg. 2012; 23(4): 1169-1173, doi: 10.1097/ SCS.0b013e31824f658a, indexed in Pubmed: 22801117.

43. Lee UY, Nam SH, Han SH, et al. Morphological characteristics of the infraorbital foramen and infraorbital canal using three-dimensional models. Surg Radiol Anat. 2006; 28(2): 115-120, doi: 10.1007/s00276-005-0071-y, indexed in Pubmed: 16432643.

44. Leo JT, Cassell MD, Bergman RA. Variation in human infraorbital nerve, canal and foramen. Ann Anat. 1995; 177(1): 93-95, doi: 10.1016/S0940-9602(11)80139-1, indexed in Pubmed: 7872502.

45. Lokanayaki V. Anatomic variations of infra orbital foramen. J Surg. 2013; 2(2): 30-36.

46. Macedo V, Cabrini R, Faig-Leite H. Infraorbital foramen location in dry human skulls. Braz J Morphol Sci. 2009; 26(1): 35-38.

47. Mailleux P, Desgain O, Ingabire MI. Ectopic infraorbital nerve in a maxillary sinus septum: another potentially dangerous variant for sinus surgery. JBR-BTR. 2010; 93(6): 308-309, indexed in Pubmed: 21381529.

48. Maravolo M, Guerra-Narducci V, Mucciolo A, et al. [Local analgesia and infraorbital block in rhinoplasty operations. Our experience]. Minerva Anestesiol. 1991; 57(9): 526-527, indexed in Pubmed: 1798469.

49. McQueen CT, DiRuggiero DC, Campbell JP, et al. Orbital osteology: a study of the surgical landmarks. Laryngoscope. 1995; 105(8 Pt 1): 783-788, doi: 10.1288/00005537-19950800000003, indexed in Pubmed: 7630287.

50. Michalek P, Donaldson W, McAleavey F, et al. Ultrasound imaging of the infraorbital foramen and simulation of the 
ultrasound-guided infraorbital nerve block using a skull model. Surg Radiol Anat. 2013; 35(4): 319-322, doi: 10.1007/ s00276-012-1039-3, indexed in Pubmed: 23129265.

51. Molliex S, Navez M, Baylot $D$, et al. Regional anaesthesia for outpatient nasal surgery. Br J Anaesth. 1996; 76(1): 151-153, indexed in Pubmed: 8672358.

52. Moore KL, Dalley AF, Agur AM. Clinically oriented anatomy. 7th Ed. Philadelphia, Lippincott Williams \& Wilkins. 2013.

53. Ozer MA, Govsa F, Kazak Z, et al. Redesign and treatment planning orbital floor reconstruction using computer analysis anatomical landmarks. Eur Arch Otorhinolaryngol. 2016; 273(8): 2185-2191, doi: 10.1007/s00405-015-3741-3, indexed in Pubmed: 26242253.

54. Peltomaa J, Rihkanen H. Infraorbital nerve recovery after minimally dislocated facial fractures. Eur Arch Otorhinolaryngol. 2000; 257(8): 449-452, indexed in Pubmed: 11073197.

55. Prabhu KP, Wig J, Grewal S. Bilateral infraorbital nerve block is superior to peri-incisional infiltration for analgesia after repair of cleft lip. Scand J Plast Reconstr Surg Hand Surg. 1999; 33(1): 83-87, indexed in Pubmed: 10207969.

56. Przygocka A, Jędrzejewski K, Szymański J, et al. Infraorbital groove localisation for the endoscopic decompression of the orbit in Graves' disease. Folia Morphol. 2015; 74(1): 78-83, doi: 10.5603/FM.2015.0013, indexed in Pubmed: 25792400.

57. Przygocka A, Podgórski M, Jędrzejewski K, et al. The location of the infraorbital foramen in human skulls, to be used as new anthropometric landmarks as a useful method for maxillofacial surgery. Folia Morphol. 2012; 71(3): 198-204, indexed in Pubmed: 22936558.

58. Przygocka A, Szymański J, Jakubczyk E, et al. Variations in the topography of the infraorbital canal/groove complex: a proposal for classification and its potential usefulness in orbital floor surgery. Folia Morphol. 2013; 72(4): 311-317, indexed in Pubmed: 24402752.

59. Rahman M, Richter EO, Osawa S, et al. Anatomic study of the infraorbital foramen for radiofrequency neurotomy of the infraorbital nerve. Neurosurgery. 2009; 64(5 Suppl 2): 423-427, doi: 10.1227/01.NEU.0000336327.10368.79, indexed in Pubmed: 19404120.

60. Rai AR, Rai R, Vadgaonkar R, et al. Anatomical and morphometric analysis of accessory infraorbital foramen. J Craniofac Surg. 2013; 24(6): 2124-2126, doi: 10.1097/ SCS.0b013e31828f2fa6, indexed in Pubmed: 24220421.

61. Raschke R, Hazani R, Yaremchuk MJ. Identifying a safe zone for midface augmentation using anatomic landmarks for the infraorbital foramen. Aesthet Surg J. 2013; 33(1): 13-18, doi: 10.1177/1090820X12468752, indexed in Pubmed: 23220876.

62. Robinson S, Wormald PJ. Patterns of innervation of the anterior maxilla: a cadaver study with relevance to canine fossa puncture of the maxillary sinus. Laryngoscope. 2005; 115(10): 1785-1788, doi: 10.1097/01.mlg.0000176544.72657.a6, indexed in Pubmed: 16222195.

63. Rontal E, Rontal M, Guilford FT. Surgical anatomy of the orbit. Ann Otol Rhinol Laryngol. 1979; 88(3 Pt 1): 382-386, doi: 10 $.1177 / 000348947908800315$, indexed in Pubmed: 464532.

64. Rusu MC, Săndulescu M, llie OC. Infraorbital canal bilaterally replaced by a lateroantral canal. Surg Radiol Anat. 2015; 37(9): 1149-1153, doi: 10.1007/s00276-015-1468-x, indexed in Pubmed: 25823691.
65. Saeedi OJ, Wang H, Blomquist PH. Penetrating globe injury during infraorbital nerve block. Arch Otolaryngol Head Neck Surg. 2011; 137(4): 396-397, doi: 10.1001/ archoto.2010.239, indexed in Pubmed: 21242530.

66. Saylam C, Özer MA, Bilge $O$, et al. Anatomic variations of the infraorbital foramen. Ann Plast Surg. 1999; 43(6): 613-617.

67. Sinanoglu A, Orhan K, Kursun S, et al. Evaluation of optic canal and surrounding structures using cone beam computed tomography: considerations for maxillofacial surgery. J Craniofac Surg. 2016; 27(5): 1327-1330, doi: 10.1097/ SCS.0000000000002726, indexed in Pubmed: 27391501.

68. Singh R. Morphometric analysis of infraorbital foramen in Indian dry skulls. Anat Cell Biol. 2011; 44(1): 79-83, doi: 10.5115/acb.2011.44.1.79, indexed in Pubmed: 21519552.

69. Song WC, Kim SH, Paik DJ, et al. Location of the infraorbital and mental foramen with reference to the soft-tissue landmarks. Plast Reconstr Surg. 2007; 120(5): 1343-1347, doi: 10.1097/01.prs.0000279558.86727.5a, indexed in Pubmed: 17898610.

70. Standring S. Gray's anatomy: the anatomical basis of clinical practice. 41th Ed. Edinburgh, Elsevier Health Sciences. 2016.

71. Takahashi $Y$, Kakizaki H, Nakano T. Infraorbital foramen: horizontal location in relation to ala nasi. Ophthalmic Plast Reconstr Surg. 2011; 27(4): 295-297, doi: 10.1097/ IOP.0b013e3182078e72, indexed in Pubmed: 21386744.

72. Taşpınar Ç. Multidetektör Bilgisayarlı Tomografi ile Değerlendirilen Canlı Kişilerde Foramen Infraorbitale Varyasyonları: Afyon Kocatepe Üniversitesi, Sağlık Bilimleri Enstitüsü, Yüksek Lisans Tezi, 46 Sayfa, Afyon. (Prof. Dr. Ahmet Songur): 2013.

73. Tezer M. Foramen infraorbitale'nin anatomik ve morfometrik özellikleri: İstanbul Üniversitesi, İstanbul Tıp Fakültesi, Uzmanlık Tezi, 44 Sayfa, İstanbul, (Prof. Dr. Adnan Öztürk. 2008.

74. Tomaszewska IM, Zwinczewska H, Gładysz T, et al. Anatomy and clinical significance of the maxillary nerve: a literature review. Folia Morphol. 2015; 74(2): 150-156, doi: 10.5603/ FM.2015.0025, indexed in Pubmed: 26050800.

75. Ukoha UU, Umeasalugo KE, Udemezue OO, et al. Anthropometric measurement of infraorbital foramen in south-east and south-south Nigeria. Natl J Med Res. 2014; 4: 225-227.

76. von Arx T, Lozanoff S, Sendi P, et al. Assessment of bone channels other than the nasopalatine canal in the anterior maxilla using limited cone beam computed tomography. Surg Radiol Anat. 2013; 35(9): 783-790, doi: 10.1007/s00276013-1110-8, indexed in Pubmed: 23539212.

77. Vriens JP, van der Glas HW, Bosman F, et al. Information on infraorbital nerve damage from multitesting of sensory function. Int J Oral Maxillofac Surg. 1998; 27(1): 20-26, indexed in Pubmed: 9506294.

78. Xu H, Guo Y, Lv D, et al. Morphological structure of the infraorbital canal using three-dimensional reconstruction. J Craniofac Surg. 2012; 23(4): 1166-1168, doi: 10.1097/ SCS.0b013e31824dfcfd, indexed in Pubmed: 22801116.

79. Yenigun A, Gun C, Uysal II, et al. Radiological classification of the infraorbital canal and correlation with variants of neighboring structures. Eur Arch Otorhinolaryngol. 2016; 273(1): 139-144, doi: 10.1007/s00405-015-3550-8, indexed in Pubmed: 25673024. 